Dept. for Speech, Music and Hearing Quarterly Progress and Status Report

\title{
The use of speech synthesis in exploring different speaking styles
}

Granström, B.

journal: STL-QPSR

volume: 32

number: $2-3$

year:

1991

pages: $\quad$ 001-010 



\title{
THE USE OF SPEECH SYNTHESIS IN EXPLORING DIFFERENT SPEAK- ING STYLES*
}

\author{
Björn Granström
}

\begin{abstract}
The possibility to vary speaker type and speaking style will be a feature of the next generation text-to-speech systems. Already today, the need for these possibilities is apparent, in dialogue systems and when speech synthesis is used as prostheses for persons with a communication handicap. Much of the information needed is not yet available. In this contribution we argue that speech synthesis itself is an efficient tool to study and understand the variability in speech. Some different methods are reviewed, representing both analysis/synthesis techniques and signal manipulations as well as text-to-speech. The main emphasis is, on the work at KTH, to study the variation of speaker and speaking styles in the context of our text-tospeech system.
\end{abstract}

\section{INTRODUCTION}

As speech synthesis technology is maturing, the demand on quality and functionality is increased. In many present and future applications it is not enough for the synthetic speech to be merely intelligible. Variation in speech, similar to the variability found in natural speech, will be requested to match many applications and to compete with alternative solutions, like huge prerecorded message banks. The range of applications asks for a variation close to the one found in human speakers. Reading stock quotations, weather reports, electronic mail, or warning messages are examples where humans would choose rather different styles of reading. In future translating speech-tospeech systems, like translating telephony (Abe, Shikano, \& Kuwabara, 1990), ideally the speaker characteristics as well as the speaking style should be preserved in the translation.

The most common application today is in aids for the handicapped. Visually impaired persons have several distinctively different demands on the function of a text-to-speech device (Carlson \& Granström, 1986). On one extreme end of the style continuum, they want hypercorrect speech that gives them maximally exact information about how the text is written. Spelling is in this case a possibility, but it is too slow and gives hardly any understanding of the text content. The other extreme is very fast speech for information scanning, something that we will report on below.

Using the speech synthesis device as a speech prosthesis, implies the general need of variation that any human feels. There is, however, a problem to make the variation convenient to use by the handicapped person in this case, since communication speed is the primary concern. Much of the variation found in natural speech is not controlled deliberately, at least not at a very conscious level. The possibility has been discussed to connect, e.g., the degree of emphasis to some myoelectric signal rather than controlling it with special symbols in the text. To our knowledge, this has not yet been successfully tried.

Apart from these practical needs in text-to-speech systems there is the scientific interest to formulate our understanding of human speech variability in explicit models. In this presentation, we will not primarily focus on the practical applications of speech synthesis, but advocate the use of speech synthesis as an exploratory tool in studying inter- and intra-speaker variability.

The need to understand variability in the speech signal is an everlasting theme in speech research. It is of equal importance for the two main strands of speech technology, speech generation and speech understanding (Carlson, 1991). The same type of knowledge is needed both in speech

*This paper was presented as an invited tutorial lecture to the ESCA workshop, "Phonetics and Phonology of Speaking Styles: Reduction and Elaboration in Speech Communication", Barcelona, Spain, Sept. 30 Oct. 2, 1991 
synthesis and speech recognition. As an example, improved production models are now being used in speech recognition experiments (Blomberg, 1990).

Large speech databases are necessary to study the wide variation in natural speech. When it comes to generalizing the observations and to test characterization of speakers and description of speaking styles, different methods of speech synthesis and speech manipulation form an important part of our tool box.

\section{EARLIER STUDIES}

\section{Different techniques}

Already with the invention of speech production systems, experiments were carried out to explore and demonstrate the capabilities to synthesize different speakers and speaking styles. An early example is Strevens (1958), who demonstrated several speaking styles, including "drunken speech" with the PAT formant synthesizer. Many researchers have used the possibility in different speech analysis/synthesis schemes to separate different aspects of the acoustic signal. Ulldal (1960) used a channel vocoder to study how changes in the intonation contour affected the perceived attitude in natural sentences. Others have followed the same track, using different techniques. Brown, Strong, \& Rechner (1974) reported on an FFT (Fast Fourier Transform) technique used to manipulate speaking rate and the mean and variance of the fundamental frequency. In this case, the objective was a "personality" rating. LPC techniques have later been widely used as a simplistic model to separate voice source and vocal tract information, primarily for prosody experiments. Traunmüller \& Branderud (1989) have used this method also for paralinguistic voice transformation experiments, manipulating both the source and the vocal tract filter. Different versions of the PSOLA method (Charpentier \& Moulines, 1989) is today the preferred analysis/synthesis technique.

Different techniques, not based on resynthesis, have been used to study similar problems. Examples are the low-pass filtering techniques (Ross, Duffy, Cooker, \& Sargeant, 1973) used to study, how the emotional content of speech is preserved in situations of severe hearing loss or only tactile speech perception. This study has been followed up by Öster \& Risberg (1986) with Swedish hard-of-hearing and normal-hearing subjects. Fonagy (1978) used the Fourcin Laryngograph as a different way of reducing the segmental information in studying emotions in speech.

\section{Experiments using text-to-speech techniques}

At CNET, different speaking styles have been modelled in their diphone-based text-to-speech system (Sorin, Larreur, \& Llorca, 1987). This experiment focussed on two different kinds of intonation models, one for a neutral "reading" style and one for a "commercial" style, used by "radio or TV speakers for news or commercial announcements". In this experiment, it was hard to include possible segmental variations, apart from duration, due to the diphone set. The possibility to create an alternative set of diphones was not used in this study, but has been part of the CNET project in supplying, e.g., male and female voices.

\section{Style experiments using a commercial synthesizer}

A large number of studies have been devoted to the acoustic correlates of different emotional states. Up to very recently the accumulated knowledge has not been used in speech synthesis. Murray, Arnott, \& Newell (1988) reports on preliminary experiments with DECtalk to create six different emotions by modifying both global settings, like speech rate, and average pitch and emotion specific "prosodic rules" for primarily segment duration and pitch contours. Cahn (1990) has reported on similar experiments, also using DECtalk. She has created a so-called "affect editor", where combinations of user-accessible parameters are used to create different speaker affects or attitudes. Applications in speaking prostheses are envisaged, but the general need in a wider context is also observed. 


\section{FULLY PROGRAMMABLE TEXT-TO-SPEECH}

The experiments using DECtalk show that even commercially available synthesizers to some extent lend themselves to experiments with style variations. Parametric synthesizers, like the one in DECtalk, have considerable flexibility compared to, e.g., a standard LPC based diphone system. In some of the products based on formant synthesizers, rudimentary voice variation is one feature. In such systems there are several possibilities to vary the speech. On the global level there are possibilities to vary speech tempo, amplitude, and voice parameters like amount of aspiration, mean pitch and pitch dynamics, vocal tract length, general speed of articulation, etc. Some of these parameters have been combined to define different voices similar also to female or child voices. The simulations of such voices are, however, still not very convincing. As a research tool more flexibility is requested, both from the production model itself and the detailed control of the associated parameters. Especially the voice source model has been improved in several systems (Carlson, Granström, \& Karlsson, 1990; Klatt \& Klatt, 1990). Our own expansion of the classical OVE III synthesizer, now named GLOVE, can form an example.

In the rule components, all sorts of effects could be modelled, i.e., as long as they are rule governed. The conditioning factors for these rules could either be given as analysis by the system, such as syntax or some measure of predictability of words, as commands to the system, or it could be given as extra information in the input text. One example of the latter is the emphasis control in the present system. By adding a number before a word in the phonetic string we can over-rule such things as default sentence stress assignment and function word reduction and also force different degrees of emphasis.

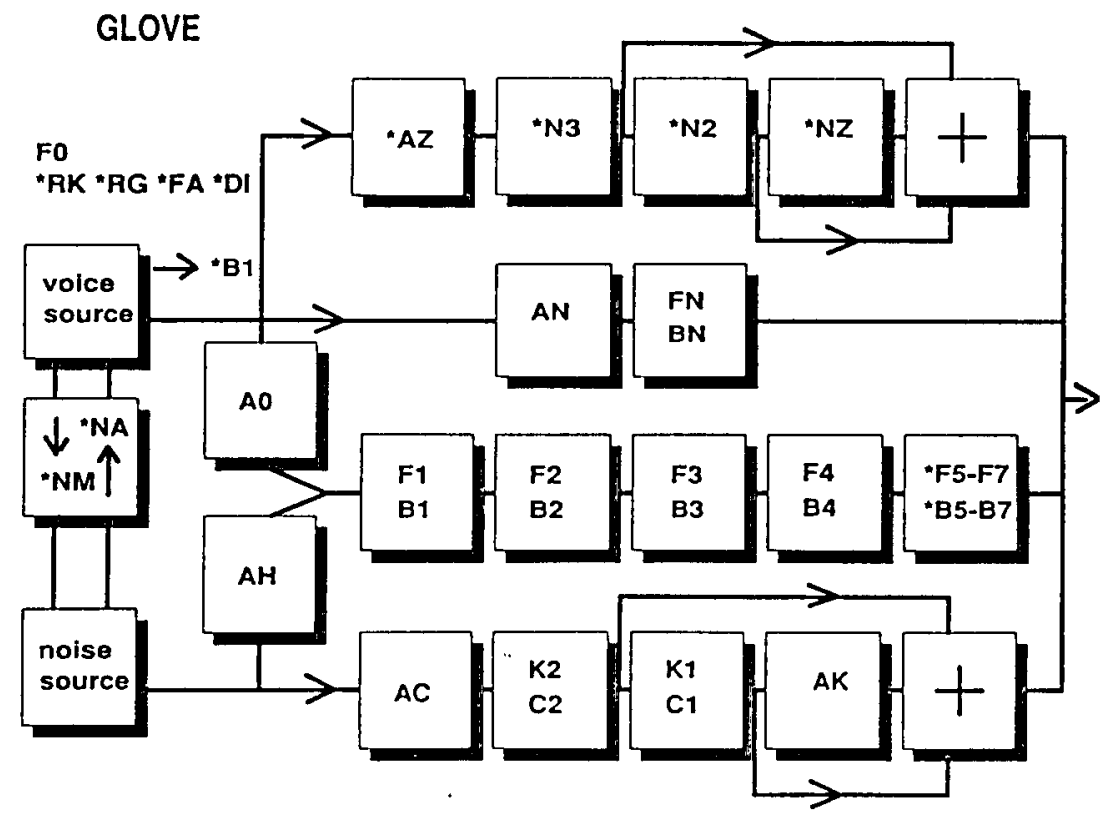

Fig. 1. Configuration of the extended synthesizer GLOVE. The new parameters are marked by *. For explanations of the parameters, see the text.

\section{AN IMPROVED PRODUCTION MODEL}

The present configuration of the GLOVE production model can be seen in Fig. 1, where the new parameters are indicated by asterisks. These are the basic parameters only. Higher order parameters, along the lines suggested by Stevens \& Bickley (1990) could be defined to make the control of the model more intuitive.

The implementation of the voice source is an expanded LF-model (Fant, Liljencrants, \& Lin, 1985). The control parameters used for this source are RK, RG, EE, FA, and F0. RK corresponds to 
the quotient between the time from peak flow to excitation and the time from zero to peak flow, this corresponds to the time from the zero-crossing to the negative peak divided by the time from the start to the zero-crossing. $R G$ is the time of the glottal cycle divided by twice the time from zero to peak flow. RG and RK are expressed in percent. EE is the excitation strength in $\mathrm{dB}$ and $\mathrm{FA}$ the frequency above which an extra $-6 \mathrm{~dB}$ /octave is added to the spectral tilt. RG and RK influence the amplitudes of the two to three lowest harmonics, FA the high-frequency content of the spectrum, and EE the overall intensity.

The synthesizer has been supplemented with a flow control of the noise source. The glottal flow waveform is used to amplitude modulate the noise. This source is used to model frication at supraglottal constrictions in the vocal tract. It is connected to the vowel branch and the parallel pole zero branch used for the synthesis of fricatives. One major advantage of the flow modulated noise is the more convincing way to synthesize voiced fricatives. The degree of this modulation can be controlled by the parameter NM.

A new parameter NA has been introduced to control the mixing of noise into the voice source. The noise is added according to the glottal opening and is thus pitch synchronous as is the noise control by the NM parameter. The NA parameter models noise generated at the glottis, while NM describes supra-glottal noise generation. Either the noise source is flow modulated (NM), or noise is added to the voice source (NA). Similar methods were also used in the source model that we explored in earlier experiments (Rothenberg, Carlson, Granström, \& Lindqvist-Gauffin, 1975).

Another vocal source parameter of a slightly different function is the DI parameter with which creak, laryngalization, or diplophonia can be simulated. We have adopted a strategy discussed in the paper by Klatt \& Klatt (1990), where every second pulse is lowered in amplitude and shifted in time.

The source/vocal-tract interaction is not implemented in the current synthesizer. The only interaction control concerns the bandwidth of the first formant that is adjusted according to glottal opening. The inclusion of this feature seems to add to the naturalness of the speech in the synthesis experiments performed.

\section{SOME EXPERIMENTS USING THE KTH RULE SYNTHESIS}

The KTH rule-synthesis system (Carlson \& al., 1990) has been used as a test bed for experiments with different kinds of voices and speaking styles. Findings from voice-source studies have been verified using the GLOVE synthesis model (Carlson \& al., 1990; Gobl \& Karlsson, 1989; Karlsson, 1988 ; 1990). Karlsson (1991) has reported on experiments on female voice description and synthesis.

The special speaking styles of a number of deaf speakers have been modelled in a study by Öster (1985). The rationale behind this study was to explore a method to demonstrate the effects of systematic speech errors on arbitrary texts and to give a means to assess the impact of different training strategies by selectively remove or change some error patterns. Similar techniques could be used in planning second language teaching.

F0 and duration have an undisputable effect on prosody but the role of intensity has been less clear. This has resulted in an emphasis on the former attributes in current speech synthesis schemes. In a recent paper (Granström \& Nord, 1991a), we have explored the use of speech intensity and also other segmental correlates of prosody. Intensity has a dynamic aspect, discriminating emphasized and reduced stretches of speech. A more global aspect of intensity must be controlled when we try to model different speaking styles. Specifically, we have been trying to model the continuum from soft to loud speech.

\section{Experiments with speaking styles for British English}

Although the concept of a multi-lingual text-to-speech system is a familiar one, rather little attention has been given to the question of the variety of each language that is synthesized. Language variation is currently being incorporated into our system both as concerns dialect and style (Bladon, Carlson, Granström, Hunnicutt, \& Karlsson, 1987). 
Our British text-to-speech implementation has been extended to provide a "style variable", a user-set range of ten values. This device can be used, for example, to propagate more affrication with a "lower" style number. The area of the system in which we first explored this style variable was in fact that of the forty or so function words ('can, have, for, them' etc.) of British English whose pronunciation, though not their spelling, varies considerably with sentence context and style. As an example, the word 'can' in a phrase 'I can go' may have a large number of realizations, some of which may be just acoustically specifiable subtleties, but some at least of which can be rendered transcriptionally: [kæn, kən, kan , kn , ? $]$ ].

It is probably reasonable to rank these forms from left to right as graded from most formal to casual. They can therefore be synthesized with style variable values of say $9,7,5,3$, and 1 , respectively. There are doubts whether style is possible to model on a one-dimensional variable, especially for an entire utterance. Style may also change within the utterance and there may well be style variants that are lexically, and individually, determined. An illustration of the simple approach can be seen in Fig. 2. The three utterances are controlled by the same phonetic string, and interestingly there is no rule specifically concerned with speech rate. The faster speech of the "casual" style is due primarily to the greater reductions allowed.

To undertake this style ranking more widely through English phonetics is, in the present state of knowledge, rather an uncertain exercise. The normative data have hardly been collected at all. At the same time, there are two particularly good motives for pressing ahead. One is that, at present, the text-to-speech developer is faced with some uncomfortable decisions of simplification when specifying such a highly variable word as "can". Another reason is a research issue.

\section{"Super human" speech rates}

In some applications the only possible way to proceed is to use the exploratory synthesis technique. Such an example is when we want to synthesize speech at super human speaking rates. Even if there are means of varying the speaking rate in the normal text-to-speech system, they are not appropriate for the extremely high speaking rates demanded by the visually impaired. Normal speaking rate is often estimated to be around 150 words per minute (wpm). This measure will be language and text dependent and it will also increase if pauses are not included. The demand from the blind is to obtain speaking rates of around $500 \mathrm{wpm}$ to approximate fast silent reading by sighted persons. One way to obtain these super human rates would be to ignore progressively more of the low information content of the text. This would mean some kind of keywords reading without any language structure. After preliminary tests we abandoned this idea. One problem with this solution was how to predict which words are keywords. It also seemed to us that the lack of linguistic structure was very confusing. If at all possible, it is our conviction that we should model the speech on human performance.

At these high speeds, however, there is no good human template. We don't want to extrapolate from the point where human speech production breaks down.

The rationale in the present attempt to increase speed further is that the phonetic component could be changed for a reduced and faster component at run-time appropriate for very high speaking rates. Especially prosodic rules are simplified or taken away. The differences between stressed and unstressed syllables are still marked by duration and fundamental frequency, a new set of inherent durations are established, and mean pitch is increased. To most listeners, the speech is close to unintelligible at this speed, though it is claimed to be useful by experienced blind listeners. In Fig. 3 two reading speeds are shown.

\section{PRELIMINARY EXPERIMENTS WITH SPEAKER ATTITUDES}

We have recently started experiments on acoustic correlates of speaker attitudes and emotions (Granström \& Nord, 1991b). As a complement to acoustic analysis, we use an exploratory synthesis method where we ask the listener to adjust presented stimuli to some internal reference, such as joy, anger, etc. 

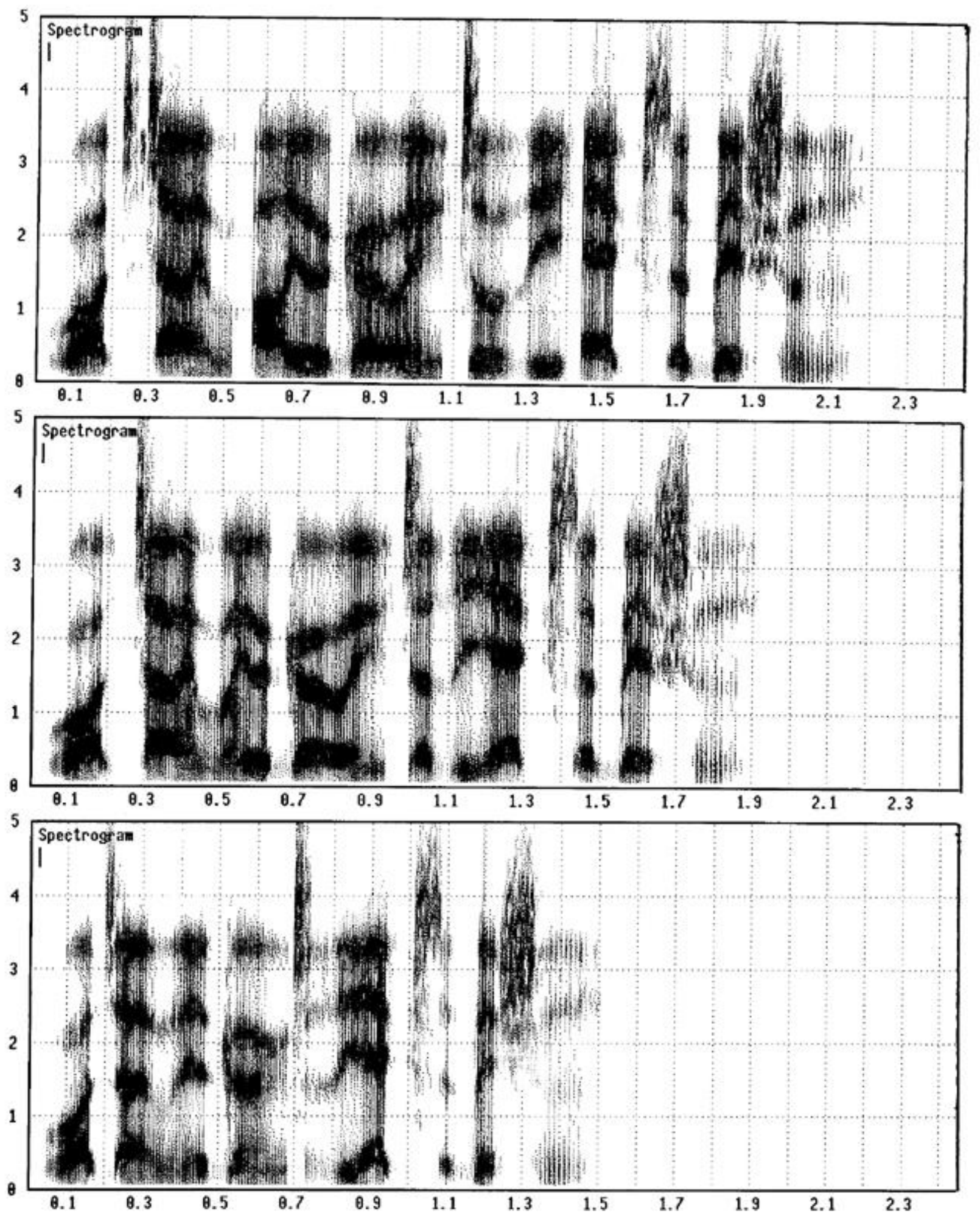

Fig. 2. The synthesized sentence "What time are you going to the exhibition" spoken in "formal" (top), "normal" (middle), and "casual" (bottom) speaking style. 

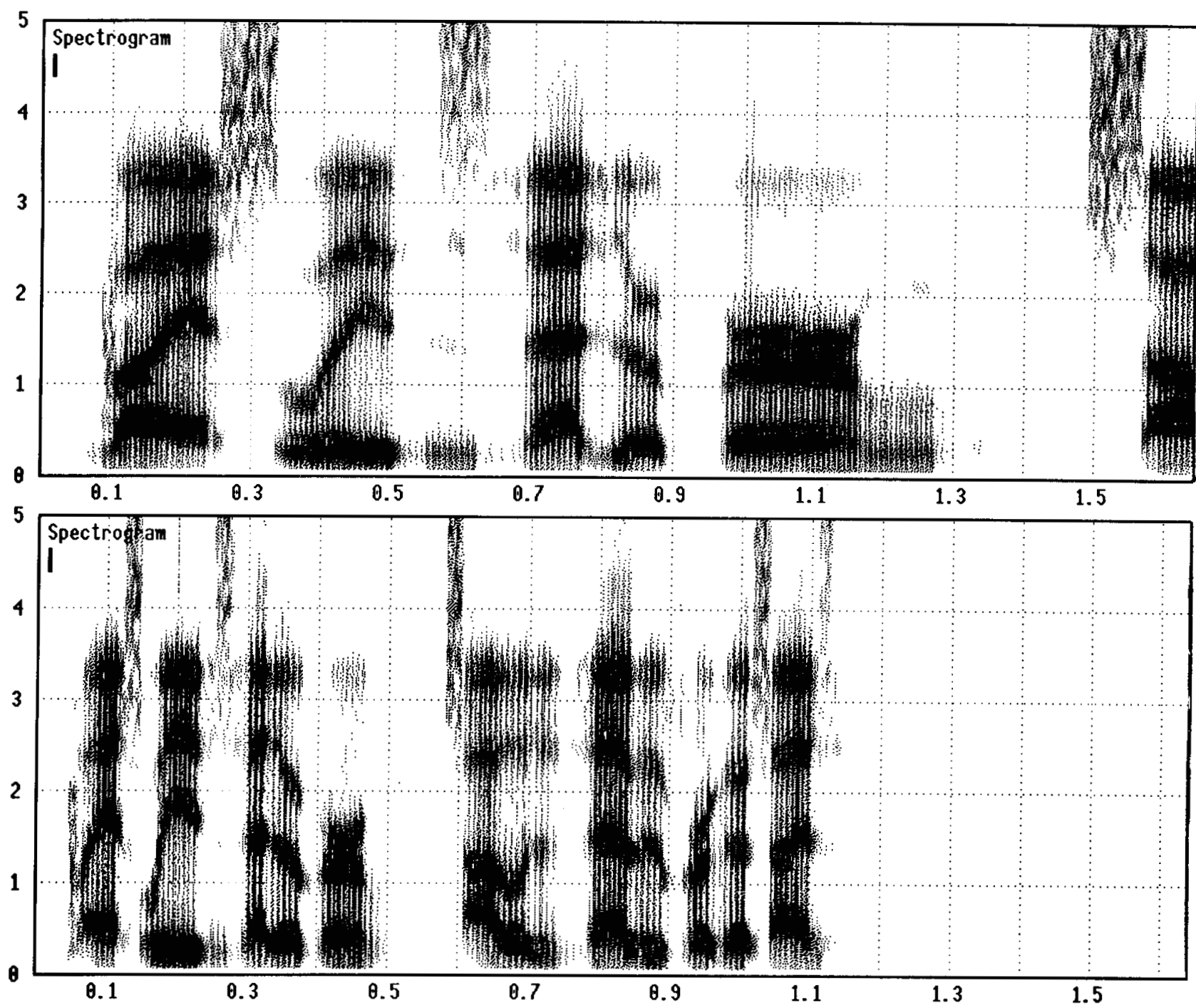

Fig.3. Spectrogram of the synthesized sentence "Buy swedes that are firm, solid, and heavy for their size" at normal speaking rate (150 wpm, truncated) and at very high rate (500 wpm).

The amount of interaction between the emotive speech and the linguistic content of a sentence is difficult to ascertain, but has to be taken into account. It is not easy to define a speech corpus that is neutral in the sense that any emotion could be combined with any of the sentences. Also some sexrelated differences might be observed. In a study by Öster \& Risberg (1986), female joy and fear were more easily confused than for male voices, where instead joy and anger were more often confused by young listeners. Furthermore, concepts like joy, anger, etc. can be expressed very differently and a unique perceptual - acoustic mapping is probably not possible.

Note that the voice does not always give away the complete speaker attitude. It is often observed that misinterpretation of emotions occurs if the listener is perceiving the speech signal without reference to visual cues. Depending on the contextual references, it is thus easy to confuse anger with joy, fright with sorrow, etc.

We have analyzed readings by two actors who were portraying different emotions by reading a fixed set of sentences in different ways: with anger, joy, fear, sadness, surprise and also in a neutral tone of voice. Examples of this analysis are given in Granström \& Nord (1991b). (The same material was previously used by Öster \& Risberg, 1986.) For some of the sentences it was obvious that the two actors made use of a number of extra factors such as sighs, voice breaks and jitter, lip smacks, etc, which often contributed in a decisive way to the intended emotion. This indicates that a standard acoustic analysis of produced sentences with different emotional content, in terms of, 
e.g., duration, intensity and pitch, does not discriminate between emotions, if the speaker relies heavily on non-phonetic cues in the production.

\begin{tabular}{|c|c|c|}
\hline $\begin{array}{r}\text { voice break } \\
0\end{array}$ & happy & $\begin{array}{l}\text { content } \\
\text { elf-assertive }\end{array}$ \\
\hline worried & neutral sure & determined \\
\hline threatening & $\begin{array}{l}\text { caution } \\
\text { angry }\end{array}$ & disappointed \\
\hline unnatural & indifferent & compliant \\
\hline
\end{tabular}

Fig. 4. Example of free verbal responses in a speech synthesis production experiment with four subjects. See text for tested dimensions.

Speech synthesis is another tool to investigate some acoustic correlates of emotion. Different analysis-by-synthesis techniques show great promise in deriving data for the synthesis of different voices, styles, and emotions. Specifically, we investigated an interactive production paradigm. We asked subjects to sit at a computer terminal and change the horizontal $(\mathrm{X})$ and vertical $(\mathrm{Y})$ position of a point within a square on the screen by means of a mouse. The $X$ and $Y$ values can be used in a set of synthesis rules, changing different aspects of the voice. In this way we set up a number of rules that changed pitch deviations, intensity, dynamics or voice source parameters of a synthesized sentence. The subjects were asked to try different combinations of these parameters by moving the mouse and to report on the impression conveyed by the synthetic sentence in terms of emotional content.

In Fig. 4, results from such an experiment are shown. The $\mathrm{X}$ dimension corresponds to the slope of the F0 declination line where a low coordinate value, (left), corresponds to a rising contour and a high value, (right), corresponds to a falling contour, with the middle of the sentence kept at a constant pitch value. The Y dimension is the pitch dynamics, where the low end corresponds to small pitch movements and the top to larger local pitch excursions. The tested sentence was one used in the Öster \& Risberg study "Dom kommer på torsdag" (They will arrive on Thursday), i.e., a linguistically quite neutral statement. Obviously, the variations suggest several different attitudes to our listeners. The task appeared quite manageable to the subjects, who responded with a fair degree of consistency. We are pursuing this line of experiments further including also voice source variations.

\section{FINAL REMARKS}

In this contribution we have advocated the use of speech synthesis techniques in exploring speaker characteristics and speaking styles. Some examples of, mostly preliminary, work in this area have been given. A fully programmable text-to-speech system with an improved parametric synthesizer is the necessary base for such experiments. The rules serve also as a framework to formalize and generalize observations from natural speech. The work is still at a very preliminary stage. The presented examples from, e.g., emotive speech suggest that the described technique is useful also for other speech dimensions. Future applications of the gained knowledge will be found in the next generation of speech synthesis and speech understanding systems. 


\section{ACKNOWLEDGEMENTS}

This work has been supported by grants from The Swedish National Language Technology Programme and Swedish Telecom.

\section{References}

Abe, M., Shikano, K., \& Kuwabara, H. (1990): "Voice conversion for an interpreting telephone," pp. 40-45 in (J. Laver, M. Jack, \& A. Gardiner eds.), ESCA Workshop on Speaker Characterization in Speech Technology, CSTR, Edinburgh.

Bladon, A., Carlson, R., Granström, B., Hunnicutt, S., \& Karlsson, I. (1987): "A text-to-speech system for British English, and issues of dialect and style," pp. 55-58 in (J. Laver \& M.A. Jack, eds.), European Conf. on Speech Technology, Vol. 1, CEP Consultants Ltd., Edinburgh.

Blomberg, M. (1990): "Adaptation to a speaker's voice in a speech recognition system based on synthetic phoneme references," pp. 58-65 in (J. Laver, M. Jack, \& A. Gardiner eds.), ESCA Workshop on Speaker Characterization in Speech Technology, CSTR, Edinburgh.

Brown, B., Strong, W., \& Rencher, A. (1974): "Fifty four voices from two: the effect of simultaneous manipulations of rate, mean fundamental frequency, and variance of fundamental frequency on ratings of personality from speech," J.Acoust.Soc.Am. 55:2, pp. 313-318.

Cahn, J.E. (1990): "The generation of affect in synthesized speech," J.Am. Voice I/O Soc. 8, pp. 1-19.

Carlson, R. \& Granström, B. (1986): "Applications of a multi-lingual text-to-speech system for the visually impaired," pp. 87-96 in (P.L. Emiliani, ed.): Development of Electronic Aids for the Visually Impaired, Martinus Nijhoff/Dr. W. Junk Publ., Dordrecht.

Carlson, R. (1991): "Synthesis: modelling variability and constraints," pp. 1043-1048 in Proc. of EUROSPEECH 91, Vol. 3, Genova.

Carlson, R., Granström, B., \& Hunnicutt, S. (1990): "Multilingual text-to-speech development and applications," pp. 269-296 in (A.W. Ainsworth, ed), Advances in Speech, Hearing and Language Processing, JAI Press, London.

Carlson, R., Granström, B., \& Karlson, I. (1990): "Experiments with voice modelling in speech synthesis," pp. 28-39 in (J. Laver, M. Jack, \& A. Gardiner, eds.), ESCA Workshop on Speaker Characterization in Speech Technology, CSTR, Edinburgh.

Charpentier, F. \& Moulines, E. (1989): "Pitch-synchronous waveform processing techniques for text-tospeech synthesis using diphones," pp. 13-19 in Proc. EUROSPEECH 89, Paris, CEP Consultants Ltd., Edinburgh.

Fant, G., Liljencrants, J \& Lin, Q. (1985): "A four-parameter model of glottal flow," STL-QPSR No. 4, pp. $1-13$.

Fonagy, I. (1978): "A new method of investigating the perception of prosodic features," Lang. \& Speech 21, pp. 34-49.

Gobl, C. \& Karlsson, I. (1989): "Male and female voice source dynamics," to be publ. in Proc. of Vocal Fold Physiology Conference, Stockholm.

Granström, B. \& Nord, L. (1991a): "Neglected dimensions in speech synthesis," paper 27 in Proc. of the ESCA Workshop on The Phonetics and Phonology of Speaking Styles: Reduction and Elaboration in Speech Communication, Barcelona.

Granström, B. \& Nord, L. (1991b): "Ways of exploring speaker characteristics and speaking styles," pp. 278-281 in Proc. of XIIth ICPhS, Vol. 4, Aix-en-Provence.

Karlsson, I. (1988): "Glottal waveform parameters for different speaker types," pp. 225-231 in (W.A. Ainsworth \& J.N. Holmes, eds.) Proc. SPEECH '88, Book 1 (7th FASE Symp.), Edinburgh.

Karlsson, I. (1990): "Female voices in speech synthesis," J. Phonetics 19, pp. 111-120. 
Karlsson, I. (1991): "Modelling voice variations in female speech synthesis," paper 33 in Proc. of the ESCA Workshop on The phonetics and Phonology of Speaking Styles: Reduction and Elaboration in Speech Communication, Barcelona, Spain.

Klatt, D. \& Klatt, L. (1990): "Analysis, synthesis, and perception of voice quality variations among female and male talkers," J.Acoust.Soc.Am. 87:2, pp. 820-857.

Murray, I.R., Arnott, J.L., \& Newell, A.F. (1988): "Hamlet - simulating emotions in synthetic speech," pp. 1217-1223 in (W.A. Ainsworth \& J.N. Holmes, eds.) Proc. SPEECH '88, Book 4 (7th FASE Symp.), Edinburgh.

Ross, M., Duffy, R., Cooker, H., \& Sargeant, D. (1973): "Contribution of the lower audible frequencies to the recognition of emotions," Am.Ann. of the Deaf, pp. 37-42.

Rothenberg, M., Carlson, R., Granström, B., \& Lindqvist-Gauffin, J. (1975): "A three-parameter voice source for speech synthesis," pp. 235-243 in (G. Fant, ed.) Speech Communication, Vol. 2, Almqvist \& Wiksell, Stockholm.

Sorin, C., Larreur, D., \& Llorca, R. (1987): "A rhythm-based prosodic parser for text-to-speech systems in French," pp. 125-128 in Proc. of XIth ICphS, Tallinn, Vol l.

Stevens, K. \& Bickley, C. (1990): "Constraints among parameters simplify control of Klatt formant synthesizer," J. Phonetics 19, pp. 161-174.

Strevens, P. (1958): The Specification of Speech Sounds by Means of Acoustic Parameters, Project report, Phonetics dept., Univ. of Edinburgh.

Traunmüller, H. \& Branderud, P. (1989): "Paralinguistic speech signal transformations," STL-QPSR No. 1, (Proc. Fonetik 89), pp. 63-68

Uldall, E. (1960): "Attitudinal meanings conveyed by intonation contours," Lang. \& Speech 3, pp. 223-234.

Öster, A-M (1985): "The use of a synthesis-by-rule system in a study of deaf speech," STL-QPSR No. 1, pp. 95-107.

Öster, A-M. \& Risberg, A. (1986): "The identification of the mood of a speaker by hearing impaired listeners," STL-QPSR No. 4, 79-90. 